\title{
Expression of microRNA-155-5p in patients with refractory diabetic macular edema and its regulatory mechanism
}

\author{
JUNWEN HE $^{1}$, RUI ZHANG ${ }^{1}$, SHAN WANG $^{2}$, LU XIE ${ }^{1}$, CHENGFENG YU $^{1}$, \\ TAO XU ${ }^{1}$, YANZI LI ${ }^{1}$ and TAO YAN ${ }^{1}$ \\ Departments of ${ }^{1}$ Retinal and Vitreous Diseases and ${ }^{2}$ Ophthalmic Imaging, \\ Aier Eye Hospital of Wuhan University, Wuhan, Hubei 430000, P.R. China
}

Received March 18, 2021; Accepted June 18, 2021

DOI: $10.3892 /$ etm.2021.10407

\begin{abstract}
Diabetic macular edema (DME) is the main cause of visual impairment in diabetic patients, but its pathogenesis remains unclear. The purpose of the present study was to analyze the expression of microRNA (miR)-155-5p in patients with DME and its regulatory mechanism. A total of 72 patients diagnosed with DME and 17 with idiopathic macular hole $(\mathrm{MH})$ were recruited. Among samples from patients with DME, 45 were DME and 27 were refractory DME, whereas patients with idiopathic $\mathrm{MH}$ served as the control group. Optical coherence tomography and fundus photograph analysis revealed that part of the retina in the fundus of patients with DME was thickened, with macular edema occurring simultaneously. In refractory patients with DME, macular edema was associated with bleeding and a dark cavity between retinal layers. Through reverse transcription-quantitative PCR analysis, miR-155-5p was highly expressed in the aqueous humor $(\mathrm{AH})$ and plasma of patients with DME compared with that in patients with $\mathrm{MH}$, and this was even higher in the refractory DME group. Upon analyzing patient clinical data, the difference in miR-155-5p expression in the $\mathrm{AH}$ and plasma was positively associated with disease course, body mass index, fasting blood-glucose, glycated hemoglobin, proteinuria and glycosuria. The expression of miR-155-5p was not significantly different based on hemoglobin, intraocular pressure and sex. The aforementioned results indicate that miR-155-5p might promote the development
\end{abstract}

Correspondence to: Mr. Tao Yan, Department of Retinal and Vitreous Diseases, Aier Eye Hospital of Wuhan University, 481 Zhongshan Road, Wuchang, Wuhan, Hubei 430000, P.R. China E-mail: aieryantao99@163.com

Abbreviations: DME, diabetic macular edema; OCT, optical coherence tomography; RT-qPCR, quantitative reverse transcription polymerase chain reaction; $\mathrm{NC}$, negative control; $\mathrm{AH}$, aqueous humor; BMI, body mass index; FBG, fasting blood-glucose; HbA1c, glycated hemoglobin; HB, hemoglobin; IOP, intraocular pressure; CCK-8, Cell Counting Kit-8; HRMECs, human retinal microvascular endothelial cells; VEGF, vascular endothelial growth factor

Key words: DME, microRNA-155-5p, OCT, human retinal epithelial cells, regulatory mechanism of DME. To further study the molecular mechanism, human retinal microvascular endothelial cells (HRMECs) were cultured and treated with high glucose in vitro. The results showed that miR-155-5p expression was significantly upregulated in HRMECs induced by high glucose. After inhibiting the expression of miR-155-5p, cell proliferation, angiogenesis and VEGF protein levels were significantly downregulated, whereas miR-155-5p mimics had the opposite effect. In summary, miR-155-5p is closely associated with DME and is a potential target for refractory DME treatment.

\section{Introduction}

DME is one of the most common causes of vision loss in diabetic patients $(1,2)$. The pathogenesis of DME is multi-factorial and involves multiple pathways (3), such as poor blood sugar control (4), high blood pressure (5), hyperlipidemia (6) and proteinuria (7), which eventually lead to the thickening of the central retina; if untreated in time, it will cause loss of vision. Currently, the treatment of refractory DME is still progressing slowly $(8,9)$.

MicroRNAs (miRNAs) are small non-coding RNAs that regulate biological networks by regulating gene expression. In several studies on DME, miRNAs have been found to play an important role in the pathogenesis of DME $(10,11)$. Studies have shown that miR-155-5p is overexpressed in diabetic patients (12), and can be used as a new biological indicator for the diagnosis and evaluation of diabetes (13).

Vascular endothelial growth factor A (VEGF-A) plays an important role in the occurrence of $\operatorname{DME}(2,14,15)$, and is the main angiogenesis promoter of endothelial cells mainly through its homologous receptor VEGF-R1. The upregulation of VEGF-A, inflammatory cytokines and chemokines induces pathological changes in the vascular endothelium, triggering the destruction of the blood-retinal barrier, causing fluid to penetrate into the extracellular space, which is clinically manifested as macular edema and leads to vision loss $(6,16)$. In vitro molecular studies demonstrated that miRNAs modulate VEGF to promote high-glucose-induced apoptosis (17) and angiogenesis (18) of HRMECs. Qiu et al (19) confirmed that miR-21-5p inhibitor inhibited the proliferation and angiogenesis of HRMECs induced by high glucose. miR-199a-3p inhibits the angiogenesis of HRMECs induced by high glucose 
by inhibiting VEGF (20). However, in DME, there has been no report on the effect of miR-155-5p targeting cytokines on the angiogenesis of HRMECs.

The present study aimed to evaluate the association between miR-155-5p and the clinical features of DME and refractory DME, as well as to evaluate the potential mechanism of miR-155-5p on HRMECs induced by high glucose in vitro.

\section{Materials and methods}

Materials. Antibodies against VEGF-A (cat. no. ab214424) and glyceraldehyde-3-phosphate dehydrogenase (GAPDH; cat. no. ab181602) were purchased from Abcam. The miR-155-5p mimics or inhibitors, and their negative controls, as well as primers for reverse transcription-quantitative (RT-q)PCR were synthesized by Guangzhou RiboBio Co., Ltd., and the miRNA sequences were as follows: miR-155-5p mimics, 5'-UUAAUG CUAAUCGUGAUAGGGGUU-3'; miR-155-5p negative control, 5'-GGGUUAGGUAAUGUAAUCCGUUUA-3'; miR-155-5p inhibitor, 5'-AACCCCTATCACGATTAGCATTAA-3'; miR-155-5p inhibitor negative control, 5'-CAGUACUUUUGU GUAGUACAA-3'. The primer sequences for miRNAs for the RT-qPCR assay were as follows: miR-155-5p forward, 5'-ACA CTCCAGCTGGGTTAATGCTAATCGTGATA-3'; miR-155-5p reverse, 5'-CTCAACTGGTGTCGTGGA-3'; U6 forward, 5'-CTC GCTTCGGCAGCACA-3'; and reverse; 5'-AACGCTTCACGA ATTTGCGT-3'.

Study subjects. Between April 2020 and August 2020, 72 patients diagnosed with DME were selected from the outpatient inpatients in Wuhan Aier Eye hospital, including

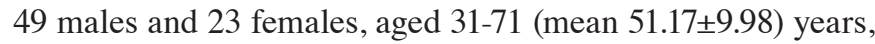
with a disease course of 0.5-20 years. All patients underwent detailed medical examinations and fundoscopy. According to the staging standard specified by the 2002 Ocular Fundus Disorders Academic Conference (21), the patients were divided into three groups as follows: 45 cases in the DME group, including 31 males and 14 females, with an average age of $50.72 \pm 11.48$ years and an average course of $6.45 \pm 1.73$ years; 27 cases in the refractory DME group, with 18 males and 9 females, average age of $53.55 \pm 9.63$ years and an average course of $9.25 \pm 3.36$ years. The exclusion criteria were as follows: Patients with diabetic ketoacidosis, diabetic hyperosmolar coma, and other acute complications of diabetes; patients with severe stress; patients with acute or chronic infections; and patients with liver disease. The patients with idiopathic macular hole (MH) (17 males and 5 females) served as the control group; their age, sex ratio and body mass index (BMI) were matched, and average age was $50.57 \pm 4.28$ years. The examination of patients with idiopathic MH excluded hypertension, heart, liver, kidney disease and other endocrine and metabolic diseases. All patients or their family members signed an informed consent form, and the experiment was approved by the Aier Eye Hospital and conformed with the guiding principles of the Declaration of Helsinki (22).

Data measurement. On admission, the patient's height and weight were measured, and BMI was calculated as weight $/$ height ${ }^{2}\left(\mathrm{~kg} / \mathrm{m}^{2}\right)$. Medical history, age, sex, course of diabetes and blood sugar control, among other parameters were recorded. Fasting blood-glucose (FBG), glycated hemoglobin (HbA1c), hemoglobin (HB), proteinuria and glycosuria were measured with the Beckman automatic biochemical analyzer (cobas c311; Roche Diagnostics GmbH) according to the kit's instructions.

Ophthalmoscopic examination. Retinal thickness was analyzed using optical coherence tomography (OCT; RTVue XR; Optovue, Inc.) using the software RTVue XR (version: 2018.1.0.43). Images of the fundus were captured using Optos PLC's Panoramic Ophthalmoscope (200TX; Nikon Corporation) with the software Optos $\mathrm{V}^{2 \oplus}$ Vantage Pro Review (version: 2.11.0.3; Nikon Corporation).

Aqueous humor (AH) sample collection and processing. $\mathrm{AH}$ was collected from each patient ( $\sim 50$ to $100 \mu \mathrm{l})$ through paracentesis. The AH samples were collected in 0.5-ml RNA-free Eppendorf (EP) tubes, stored on ice, and further processed within $1 \mathrm{~h}$ after collection. In all cases, sample collection was non-invasive, eliminating the risk of blood or cell debris contamination. By centrifuging the AH sample at $1,200 \mathrm{x} g$ for $30 \mathrm{~min}$ at $4^{\circ} \mathrm{C}$, the final cell/cell debris contamination could be prevented. Then, the supernatant was transferred to a new sterile EP tube for RNA extraction.

Collection and processing of whole blood samples. Firstly, $5 \mathrm{ml}$ whole blood was collected from the patients in a BD Vacutainer-EDTA tube (BD Biosciences) and immediately mixed 5-8 times. Within $1 \mathrm{~h}$ after blood collection, the blood samples were processed by centrifugation at $1,200 \mathrm{x} \mathrm{g}$ for $10 \mathrm{~min}$ at $4^{\circ} \mathrm{C}$; the collected plasma was centrifuged at $1,200 \mathrm{xg}$ for $20 \mathrm{~min}$ at $4^{\circ} \mathrm{C}$ to remove contaminant cells and cell debris, and then RNA extraction was performed. Hemolyzed plasma samples were excluded from the study.

Cell culture, transfection and treatments. The HRMECs (cat. no. CP-H130; Procell Life Science \& Technology Co., Ltd.) were cultured at $37^{\circ} \mathrm{C}$ with $5 \% \mathrm{CO}_{2}$ in complete culture medium (cat. no. CM-H130; Procell Life Science \& Technology Co., Ltd.). Once cells achieved 60-80\% confluence, they were transfected with RNA using Lipofectamine ${ }^{\circledR} 2000$ (Invitrogen; Thermo Fisher Scientific, Inc.) at $37^{\circ} \mathrm{C}$ for $4 \mathrm{~h}$, according to the manufacturer's instructions. Then, medium was replaced with fresh culture medium, and cells were incubated for a further $48 \mathrm{~h}$ prior to RT-qPCR or western blot analysis. The high-glucose group was pre-cultured with $30 \mathrm{mM}$ glucose at $37^{\circ} \mathrm{C}$ for $48 \mathrm{~h}$, and the normal glucose group (control) was pre-cultured with $5 \mathrm{mM}$ glucose at $37^{\circ} \mathrm{C}$ for $48 \mathrm{~h}$ before experiment. The glucose concentration used in the high glucose group was based on previous studies $(23,24)$.

RNA extraction. Using the miRNeasy Mini kit (217004; Qiagen $\mathrm{GmbH}$ ), total RNA was extracted from $50 \mu \mathrm{l} \mathrm{AH}$ or $200 \mu 1$ plasma samples. The $\mathrm{AH}$ and plasma samples were centrifuged at $3000 \mathrm{xg}$ for $5 \mathrm{~min}$ at $4^{\circ} \mathrm{C}$ to completely remove contaminant cell debris. A total of $50 \mu \mathrm{l} \mathrm{AH}$ and $200 \mu \mathrm{l}$ plasma per patient was diluted to $400 \mu 1$ with RNA-free water (Gibco; Thermo Fisher Scientific, Inc.) to avoid protein aggregation. Then, the sample was lysed by adding three times the volume of TRIzol ${ }^{\circledR}$ (Invitrogen; Thermo Fisher Scientific, Inc.) and was finally dissolved in $10 \mu 1$ RNA-free water. 

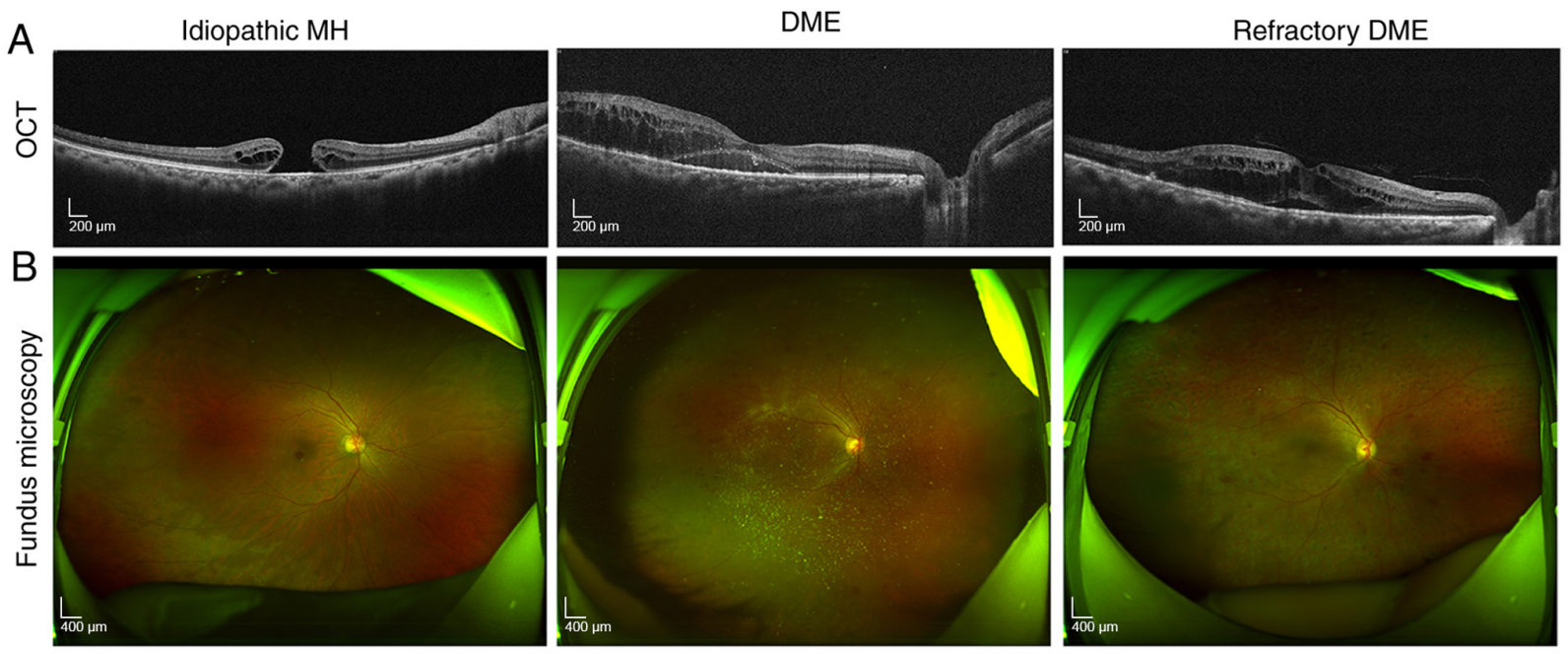

Figure 1. Refractory DME exacerbates the symptoms of macular edema. (A) OCT analyses of the retinal symptoms among patients in different groups (B) Fundus microscopy to analyze the differences in macular edema area and eyeball characteristics among patients in different groups. OCT, optical coherence tomography; DME, diabetic macular edema; MH, macular hole.

$R T$-qPCR assay. Extracted RNA was reverse transcribed to cDNA using a PrimeScript RT reagent kit (Thermo Fisher Scientific, Inc.), according to the manufacturer's instructions. The RT-qPCR procedure was performed using a SYBR Premix ExTaq II kit (Thermo Fisher Scientific, Inc.) according to the manufacturer's instructions in conjunction with a 7500 Real-Time PCR System (Applied Biosystems; Thermo Fisher Scientific, Inc.). The thermocycling conditions were as follows: $95^{\circ} \mathrm{C}$ for $10 \mathrm{~min} ; 55^{\circ} \mathrm{C}$ for $2 \min ; 72^{\circ} \mathrm{C}$ for $2 \mathrm{~min}$; followed by 40 cycles of $95^{\circ} \mathrm{C}$ for $15 \mathrm{sec}$ and $60^{\circ} \mathrm{C}$ for $1 \mathrm{~min}$. Target miR-155-5p levels were normalized to those of the housekeeping gene U6. Relative miR-155-5p expression levels were calculated using the $2^{-\Delta \Delta C q}$ method (25), and the RT-qPCR experiment was repeated three times.

Western blotting. Transfected HRMECs were harvested by trypsin digestion and lysed using ice-cold RIPA lysis buffer (Beyotime Institute of Biotechnology). Lysate protein concentrations were estimated using a BCA protein assay kit (Beijing Solarbio Science \& Technology Co., Ltd.). Equal amounts of denatured proteins $(20 \mu \mathrm{g})$ were resolved using $10 \%$ SDS-polyacrylamide gel electrophoresis (Beijing Solarbio Science \& Technology Co., Ltd.) and protein bands were transferred to a polyvinylidene fluoride membrane (Beijing Solarbio Science \& Technology Co., Ltd.). The membrane was blocked with 5\% BSA (Beijing Solarbio Science \& Technology Co., Ltd.) for $1 \mathrm{~h}$ at $22 \pm 3^{\circ} \mathrm{C}$. Subsequently, the membranes were incubated with an anti-VEGF antibody $(1: 1,000)$ overnight at $4^{\circ} \mathrm{C}$, rinsed, and incubated with the horseradish peroxidase-conjugated secondary antibody (goat anti-rabbit; 1:10,000; cat. no. ab205718; Abcam) for $2 \mathrm{~h}$ at $22 \pm 3^{\circ} \mathrm{C}$. Protein bands were visualized by the addition of ELC enhanced chemiluminescence reagent (Thermo Fisher Scientific, Inc.) in conjunction with an imaging system (DNR Bio-Imaging Systems, Ltd.). An anti-GAPDH antibody $(1: 10,000)$ was used as a loading control.

Cell Counting Kit-8 (CCK8) assay. Briefly, a single cell suspension was prepared by the trypsin method and inoculated in 96-well plates at a density of $3 \times 10^{3}$ cells/well. Then,
$10 \mu 1$ CCK-8 reagent (Elabscience, Wuhan, China) was added at $0,24,48$ and $72 \mathrm{~h}$ and incubated for $60 \mathrm{~min}$. The optical density was measured at $490 \mathrm{~nm}$ using a microplate reader (multiscan MK3; Thermo Fisher Scientific, Inc.). The CCK-8 experiment was repeated three times.

Angiogenesis assay. Transfected HRMECs were pretreated with different concentrations of glucose $(5$ or $30 \mathrm{mM})$ at $37^{\circ} \mathrm{C}$ for 48 h. Next, serum-starved HRMECs $\left(2 \times 10^{4}\right.$ cells) were seeded onto 24-well plates coated with Matrigel (BD Biosciences) in endothelial basal medium and incubated at $37^{\circ} \mathrm{C}$ with $5 \% \mathrm{CO}_{2}$ for $24 \mathrm{~h}$. Tubular structures of HRMECs in the Matrigel were examined with a light microscope (Olympus Corporation). The angiogenesis experiment was repeated three times and five fields were randomly selected for the quantitative analysis of each result.

Statistical analysis. All data are expressed as means \pm standard deviations. All statistical analyses were performed using SPSS version 21.0 statistical analysis package (SPSS Inc.). The male/female data were analyzed using a $\chi^{2}$ test. The $\mathrm{AH}$, serum and clinical characteristics of patients, the expression of miR-155-5p under miR-155-5p mimic/inhibitor transfection, cell proliferation, angiogenesis, and western blot data proceeded via a one-way analysis of variance and Bonferroni post hoc test. The difference of miR-155-5p expression before and after HG induction was analyzed using the unpaired Student's t-test. $\mathrm{P}<0.05$ was considered to indicate a statistically significant difference.

\section{Results}

Ophthalmoscopic examination. In the present study, fundus microscopy was performed on patients in each group. The results showed that compared with that in the control group, patients in the DME group had obvious macular edema and thick retina. Furthermore, compared with disease signs in the DME group, patients with refractory DME had bleeding on the eyeballs, as well as old laser spots remaining from previous treatment (Fig. 1A). 

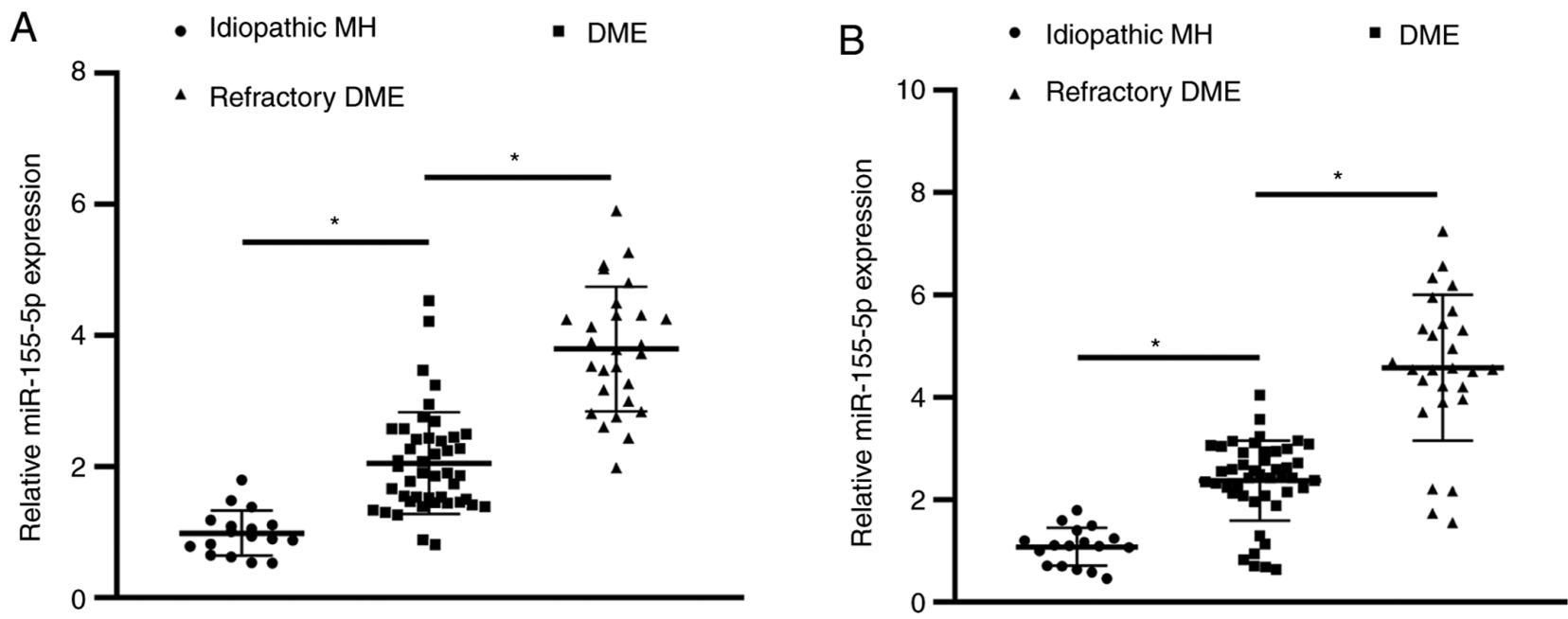

Figure 2. Differences in the expression levels of miRNA between DME and refractory DME. Reverse transcription-quantitative polymerase chain reaction analysis of the differences in the expression of miR-155-5p in the aqueous humor (A) and serum (B) of patients with DME. "P<0.05. DME, diabetic macular edema; MH, macular hole; miRNA/miR, microRNA.

Optical coherence tomography analysis. The results showed that compared with the control group, patients with DME had notable edema between the retinal layers, whereas patients with refractory DME had more obvious retinal edema. Meanwhile, patients with refractory DME have obvious edema and dark cavities between the retinal layers (Fig. 1B).

Differences in the expression of AH and serum miR-155-5p among patients with DME. Compared with patients in the control group, the expression of miR-155-5p in AH and serum of the DME group was upregulated (Fig. 2A and B). The expression of miR-155-5p in AH and serum of patients in the refractory DME group was also upregulated compared with that in patients in the DME group.

Association between the expression level of miR-155-5p and patient clinical characteristics. Next, the clinical characteristics of patients in each group were analyzed, including BMI, FBG, HbA1c, HB, intraocular pressure (IOP), proteinuria and glycosuria. The results showed that the differences in miR-155-5p expression were positively associated with the course of disease, BMI, FBG, HbA1C, proteinuria and glycosuria (Fig. 3). The expression level of miR-155-5p was not significantly different based on the age, HB, IOP and sex of the patients. Specific data are shown in Table SI.

High glucose induces increased expression of miR-155-5p. Next, the potential mechanism underlying the effects of miR-155-5p was analyzed from a molecular perspective. For this, HRMECs were treated with high glucose in vitro. RT-qPCR results showed that high glucose induced the upregulation of miR-155-5p (Fig. 4A).

Effect of miR-155-5p on HRMECs. To confirm the effect of miR-155-5p in HRMECs, synthetic miR-155-5p mimics/inhibitors were transfected into cells and the effect on the cellular functions of HRMECs was analyzed. RT-qPCR analysis showed that miRNAs were successfully constructed (Fig. 4B). The results also showed that high glucose promotes the proliferation and angiogenesis of HRMECs and also upregulates the protein level of VEGF-A (Fig. 4C-G). These effects could be reversed by a miR-155-5p inhibitor, and at the same time, these effects were enhanced by miR-155-5p mimics. These results confirm that miR-155-5p can regulate the proliferation and angiogenesis of HRMECs and concomitantly affect the protein level of VEGF-A in HRMECs.

\section{Discussion}

DME, defined as the thickening of the retina involving or near the center of the macula, is the most common cause of vision loss in patients affected by diabetes (26). At present, intravitreal injection of anti-VEGF drugs is considered one of the best treatments for DME $(14,27)$. Early vitrectomy can also effectively decrease the burden of treatment for diabetic patients and prevent vision loss (28-31). However, for some patients with intractable DME, these treatments have not achieved long-term relief of the disease $(9,32,33)$. The present study analyzed the eyeball characteristics of patients with DME and found that the retina of patients with idiopathic MH did not have obvious edema, whereas patients with DME, in addition to more intractable patients with DME, had obvious retinal edema. Meanwhile, the retina of patients with refractory DME also had symptoms of hemorrhage, and the retina was significantly heavier compared with that of DME patients. In addition, patients with intractable DME had obvious edema and dark cavities between the omentum layers.

Studies have shown that miRNA, as a biomarker of DME, has high sensitivity and specificity (11) and is stably expressed in biological fluids such as human AH and plasma $(34,35)$. Among them, overexpression of miR-155-5p has been proved by numerous studies to play an important role in the progression of diabetes $(36,37)$. However, the study of miR-155-5p in DME has not been reported. In the present study, the role of $\mathrm{AH}$ and plasma miR-155-5p was investigated in DME. One of the main results of the present study showed that the expression level of miR-155-5p was relatively increased in the DME group and the refractory DME group compared with 

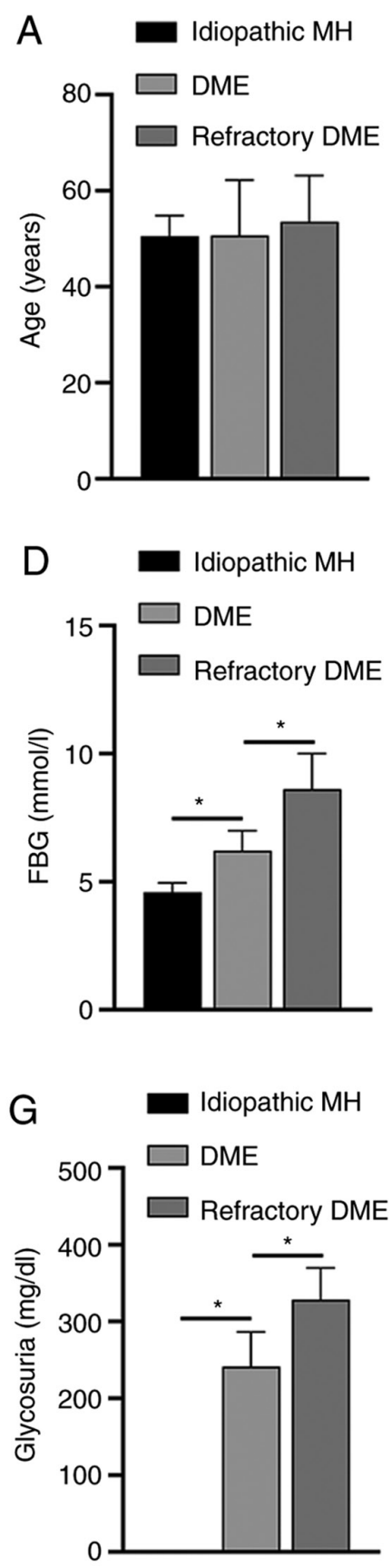
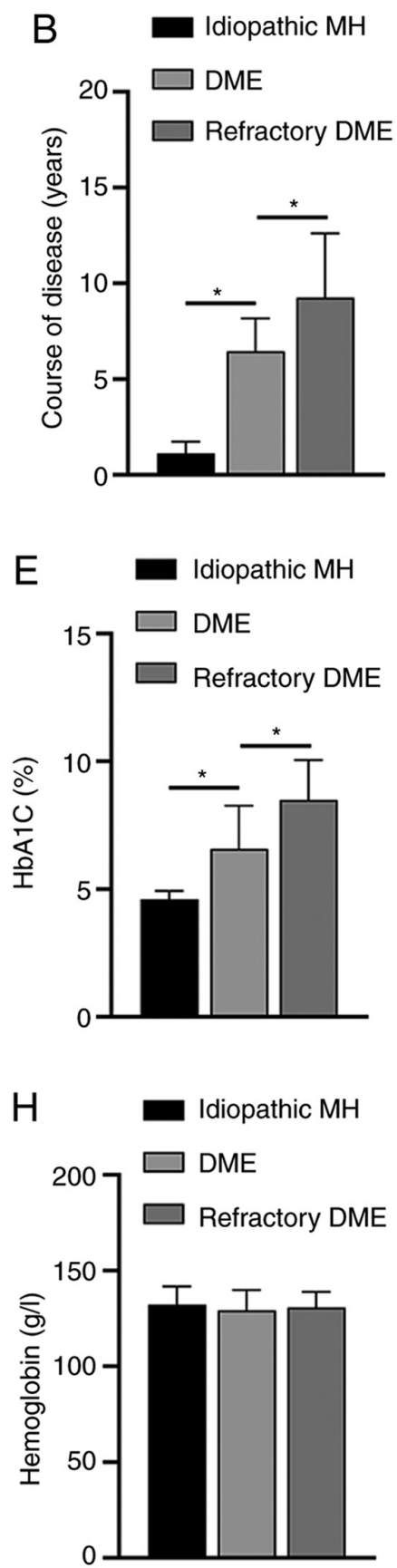

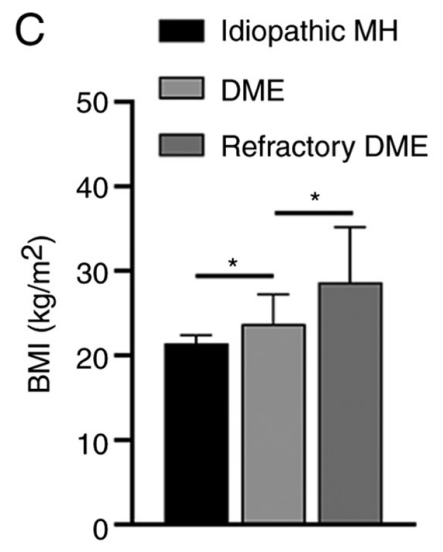

F
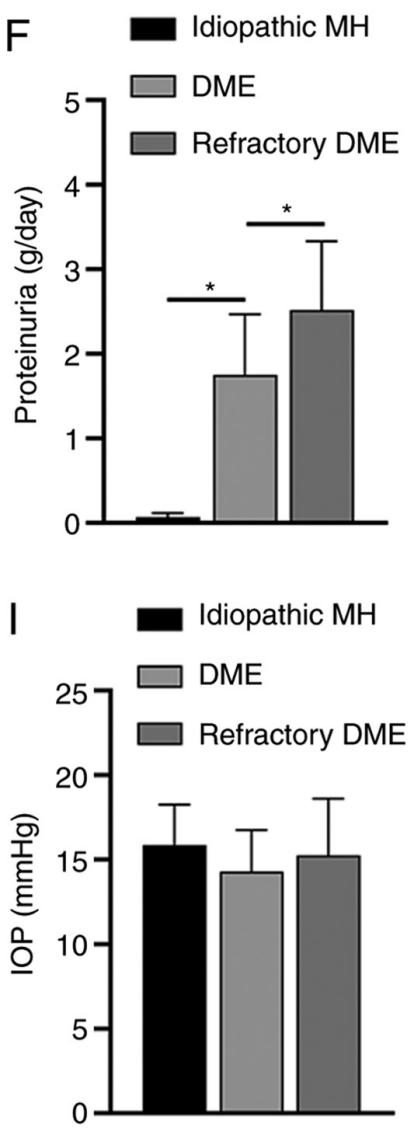

Figure 3. Association between miR-155-5p and clinical symptoms of patients. The differences in the age (A), course of disease (B), BMI (C), FBG (D), $\operatorname{HbA1C}(\mathrm{E})$, proteinuria $(\mathrm{F})$, glycosuria $(\mathrm{G}), \mathrm{HB}(\mathrm{H})$ and IOP $(\mathrm{I})$ in each group of patients are shown. ${ }^{*} \mathrm{P}<0.05$. miR, microRNA; DME, diabetic macular edema; MH, macular hole; BMI, body mass index; FBG, fasting blood-glucose; HbAlc, glycated hemoglobin; HB, hemoglobin; IOP, intraocular pressure.

that of the control group. In addition, the expression level of miR-155-5p in the refractory DME group was significantly higher compared with that in the DME group. The results thus showed that the expression level of miR-155-5p is associated with the severity of DME. Accordingly, abnormal miR-155-5p expression is associated with the development of DME, and the regulation of its expression might provide a potential treatment for this disease.

Regarding the mechanism underlying the effects of miR-155-5p on the development of DME, the association between this marker and some clinical indicators was studied. The analysis of the present study showed that the expression level of miR-155-5p was positively associated with the course of disease, BMI, FBG, HbA1C, proteinuria and glycosuria. In addition, the expression level of miR-155-5p had no obvious association with HB, IOP and sex. According to the clinical research data, it is necessary to further clarify the mechanism associated with the effects of miR-155-5p in DME. Therefore, the present study simulated hyperglycemia conditions by exposing HRMECs to high glucose; this significantly upregulated the expression of miR-155-5p in these cells. High glucose in HRMECs significantly downregulated the proliferation and angiogenesis of HRMECs and increased the level of VEGF-A protein compared with that with normal glucose treatment. However, after inhibiting the expression of miR-155-5p, the effect of high glucose was reversed. Moreover, the addition 

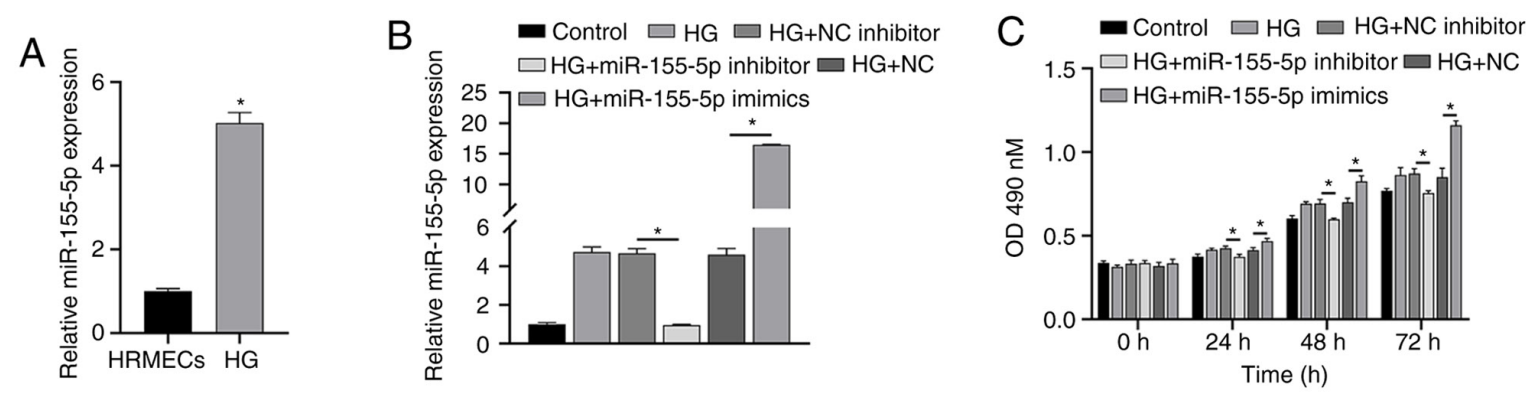

D
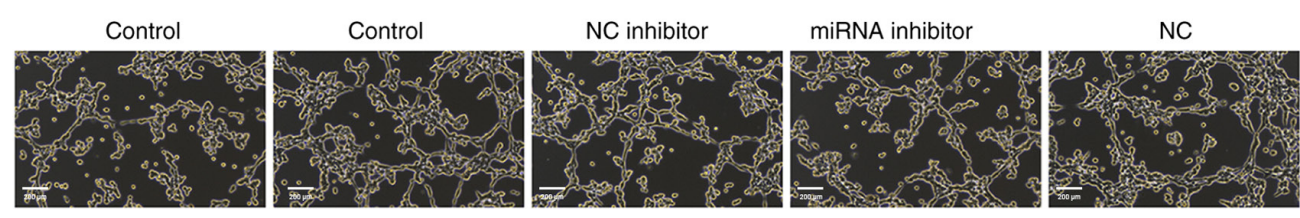

miRNA mimics
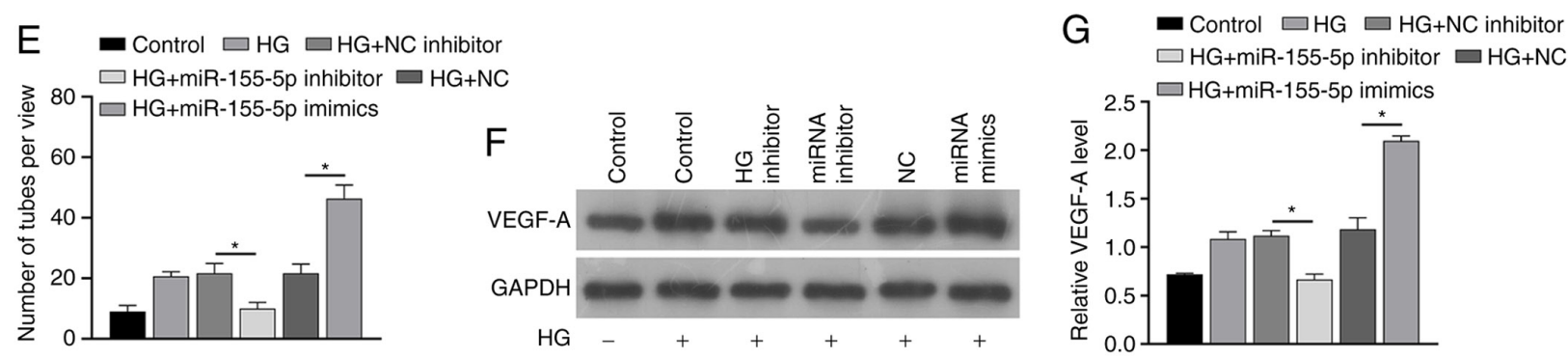

Figure 4. miR-155-5p reverses the effect of high glucose induction. RT-qPCR (A) analysis showed that the expression of miR-155-5p was different in HRMECs induced by high glucose. After transfection with miRNAs, RT-qPCR (B) and Cell Counting Kit- 8 assays (C) were performed to analyze the changes in expression of miR-155-5p and cell proliferation in each group. Angiogenesis (D and E) was analyzed in each group, and western blotting (F and G) was performed to analyze the changes in VEGF-A protein levels. Samples were divided into the control group, HG group, HG+NC inhibitor group, HG+inhibitor group, HG+NC group and HG+miR-155-5p mimic group. ${ }^{*}<0.05$. miR/miRNA, microRNA; RT-qPCR, reverse transcription-quantitative polymerase chain reaction; $\mathrm{HG}$, high glucose; NC negative control; VEGF, vascular endothelial growth factor; HRMECs, human retinal microvascular endothelial cells

of miR-155-5p mimics had the same effect as high sugar. The aforementioned results confirm that miR-155-5p can regulate the cellular function of HRMECs induced by high glucose.

The present study has limitations. Firstly, VEGF and miR-155-5p expression vectors were not injected into the vitreous of type 2 diabetes mellitus animals to verify the utility of miR-155-5p. Secondly, further research on the hypothetical target genes and associated signaling pathways of miR-155-5p were not conducted. Finally, the number of patients was insufficient to clearly describe the association between miR-155-5p and DME or intractable DME, to deepen the understanding of the field. The aforementioned deficiencies also suggest the direction of future research. Additional experiments are intended to be conducted in the future to verify the association between miRNA and DME or intractable DME.

In summary, the expression level of miR-155-5p in $\mathrm{AH}$ and plasma is upregulated during the development of DME and can be used as an indicator for this disease. The expression level of miR-155-5p is also positively associated with the course of disease, BMI, FBG, HbA1C, proteinuria and glycosuria. The present study provides a valuable reference for the diagnosis and treatment of DME and refractory DME.

\section{Acknowledgements}

Not applicable.

\section{Funding}

The present study was supported by the Science Research Foundation of Aier Eye Hospital Group (grant no. AF1901D5) and Medical Research Fund of Wuhan Municipal Health Commission (grant no. WX18D15).

\section{Availability of data and materials}

The datasets used and/or analyzed during the current study are available from the corresponding author on reasonable request.

\section{Authors' contributions}

JH and TY designed the study and developed the methodology. RZ, SW, LX, TX, CY and YL performed the experiments and collected the data. JH, TY, RZ and SW analyzed and interpreted the data. JH and TY drafted the original manuscript. $\mathrm{JH}$ and TY confirm the authenticity of all the raw data. All authors read and approved the final manuscript.

\section{Ethics approval and consent to participate}

The study was approved by the ethics committee of Wuhan Aier Eye Hospital (permit no. 2021IRBLW01). Written informed consent was obtained from each patient. 


\section{Patient consent for publication}

Not applicable.

\section{Competing interests}

The authors declare that they have no competing interests.

\section{References}

1. Tan GS, Cheung N, Simó R, Cheung GC and Wong TY: Diabetic macular oedema. Lancet Diabetes Endocrinol 5: 143-155, 2017.

2. Patelli F, Radice P and Giacomotti E: Diabetic macular edema. Dev Ophthalmol 54: 164-173, 2014.

3. Yau JW, Rogers SL, Kawasaki R, Lamoureux EL, Kowalski JW, Bek T, Chen SJ, Dekker JM, Fletcher A, Grauslund J, et al Meta-Analysis for Eye Disease (META-EYE) Study Group: Global prevalence and major risk factors of diabetic retinopathy. Diabetes Care 35: 556-564, 2012.

4. Fenwick EK, Xie J, Man REK, Sabanayagam C, Lim L, Rees G Wong TY and Lamoureux EL: Combined poor diabetes contro indicators are associated with higher risks of diabetic retinopathy and macular edema than poor glycemic control alone. PLoS One 12: e0180252, 2017.

5. Stana D, Potop V, Istrate SL, Eniceicu C, Mihalcea AR, Paşca IG, Aqel A, Ciuluvică R and Moraru D: Variability of diabetic macular edema in correlation with hypertension retinopathy in patients with diabetes mellitus and essential hypertension. Rom J Ophthalmol 63: 327-338, 2019.

6. Romero-Aroca P, Baget-Bernaldiz M, Pareja-Rios A, Lopez-Galvez M, Navarro-Gil R and Verges R: Diabetic Macular Edema Pathophysiology: Vasogenic versus Inflammatory. J Diabetes Res 2016: 2156273, 2016.

7. Shye M, Hanna RM, Patel SS, Tram-Tran N, Hou J, Mccannel C, Khalid M, Hanna M, Abdelnour L and Kurtz I: Worsening proteinuria and renal function after intravitreal vascular endothelial growth factor blockade for diabetic proliferative retinopathy. Clin Kidney J 13: 969-980, 2020.

8. Kim JE, Pollack JS, Miller DG, Mittra RA and Spaide RF; Isis Study Group: ISIS-DME: A prospective, randomized, dose-escalation intravitreal steroid injection study for refractory diabetic macular edema. Retina 28: 735-740, 2008.

9. Hussain RM and Ciulla TA: Treatment strategies for refractory diabetic macular edema: Switching anti-VEGF treatments, adopting corticosteroid-based treatments, and combination therapy. Expert Opin Biol Ther 16: 365-374, 2016.

10. Grieco GE, Sebastiani G, Eandi CM, Neri G, Nigi L, Brusco N, D'Aurizio R, Posarelli M, Bacci T, Benedetto E, et al: MicroRNA Expression in the Aqueous Humor of Patients with Diabetic Macular Edema. Int J Mol Sci 21: 21, 2020.

11. Chan HW, Yang B, Wong W, Blakeley P, Seah I, Tan QS, Wang $\mathrm{H}$, Bhargava $\mathrm{M}$, Lin $\mathrm{HA}$, Chai $\mathrm{CH}$, et al: A pilot study on MicroRNA profile in tear fluid to predict response to anti-VEGF treatments for diabetic macular edema. J Clin Med 9: 9, 2020.

12. Wang J, Wang G, Liang Y and Zhou X: Expression profiling and clinical significance of plasma MicroRNAs in diabetic nephropathy. J Diabetes Res 2019: 5204394, 2019.

13. Bai X, Luo Q, Tan K and Guo L: Diagnostic value of VDBP and miR-155-5p in diabetic nephropathy and the correlation with urinary microalbumin. Exp Ther Med 20: 86, 2020.

14. Kim EJ, Lin WV, Rodriguez SM, Chen A, Loya A and Weng CY: Treatment of diabetic macular edema. Curr Diab Rep 19: 68, 2019.

15. Lally DR, Shah CP and Heier JS: Vascular endothelial growth factor and diabetic macular edema. Surv Ophthalmol 61: 759-768, 2016.

16. Miller K and Fortun JA: Diabetic Macular Edema: Current understanding, pharmacologic treatment options, and developing therapies. Asia Pac J Ophthalmol (Phila) 7: 28-35, 2018.

17. Zeng Y, Cui Z, Liu J, Chen J and Tang S: MicroRNA-29b-3p promotes human retinal microvascular endothelial cell Apoptosis via blocking SIRT1 in diabetic retinopathy. Front Physiol 10: 1621,2020

18. Han N, Xu H, Yu N, Wu Y and Yu L: MiR-203a-3p inhibits retinal angiogenesis and alleviates proliferative diabetic retinopathy in oxygen-induced retinopathy (OIR) rat model via targeting VEGFA and HIF-1 $\alpha$. Clin Exp Pharmacol Physiol 47: $85-94,2020$
19. Qiu F, Tong H, Wang Y, Tao J, Wang H and Chen L: Inhibition of miR-21-5p suppresses high glucose-induced proliferation and angiogenesis of human retinal microvascular endothelial cells by the regulation of AKT and ERK pathways via maspin. Biosci Biotechnol Biochem 82: 1366-1376, 2018.

20. Wang L, Liu WX and Huang XG: MicroRNA-199a-3p inhibits angiogenesis by targeting the VEGF/PI3K/AKT signalling pathway in an in vitro model of diabetic retinopathy. Exp Mol Pathol 116: 104488, 2020.

21. Wilkinson CP, Ferris FL III, Klein RE, Lee PP, Agardh CD Davis M, Dills D, Kampik A, Pararajasegaram R and Verdaguer JT; Global Diabetic Retinopathy Project Group: Proposed international clinical diabetic retinopathy and diabetic macular edema disease severity scales. Ophthalmology 110: 1677-1682, 2003.

22. Mastroleo I: Post-trial obligations in the Declaration of Helsinki 2013: Classification, reconstruction and interpretation. Developing World Bioeth 16: 80-90, 2016.

23. Lu L, Lu Q, Chen W, Li J, Li C and Zheng Z: Vitamin D3 Protects against diabetic retinopathy by inhibiting high-glucose-induced activation of the ROS/TXNIP/NLRP3 inflammasome pathway. J Diabetes Res 2018: 8193523, 2018.

24. Gu C, Draga D, Zhou C, Su T, Zou C, Gu Q, Lahm T, Zheng Z and Qiu Q: miR-590-3p inhibits pyroptosis in diabetic retinopathy by targeting NLRP1 and inactivating the NOX4 signaling pathway. Invest Ophthalmol Vis Sci 60: 4215-4223, 2019.

25. Livak KJ and Schmittgen TD: Analysis of relative gene expression data using real-time quantitative PCR and the 2(-Delta Delta C(T)) method. Methods 25: 402-408, 2001.

26. Bandello F, Battaglia Parodi M, Lanzetta P, Loewenstein A, Massin P, Menchini F and Veritti D: Diabetic macular edema. Dev Ophthalmol 58: 102-138, 2017.

27. Korobelnik JF, Do DV, Schmidt-Erfurth U, Boyer DS, Holz FG, Heier JS, Midena E, Kaiser PK, Terasaki H, Marcus DM, et al: Intravitreal aflibercept for diabetic macular edema. Ophthalmology 121: 2247-2254, 2014.

28. Michalewska Z, Stewart MW, Landers MB III, Bednarski M, Adelman RA and Nawrocki J: Vitrectomy in the management of diabetic macular edema in treatment-naïve patients. Can J Ophthalmol 53: 402-407, 2018

29. Simunovic MP, Hunyor AP and Ho IV: Vitrectomy for diabetic macular edema: A systematic review and meta-analysis. Can J Ophthalmol 49: 188-195, 2014.

30. Jackson TL, Nicod E, Angelis A, Grimaccia F, Pringle E and Kanavos P: Pars Plana Vitrectomy For Diabetic Macular Edema: A Systematic Review, Meta-Analysis, and Synthesis of Safety Literature. Retina 37: 886-895, 2017.

31. Michalewska Z, Stewart MW, Landers MB III, Bednarski M, Adelman RA and Nawrocki J: Response to Vitrectomy in diabetic macular edema. Can J Ophthalmol 54: 403-404, 2019.

32. Choi MY, Jee D and Kwon JW: Characteristics of diabetic macular edema patients refractory to anti-VEGF treatments and a dexamethasone implant. PLoS One 14: e0222364, 2019.

33. Maleki A, Stephenson AP and Hajizadeh F: Topical interferon alpha $2 b$ in the treatment of refractory diabetic macular edema. J Ophthalmic Vis Res 15: 453-458, 2020.

34. Mastropasqua R, Toto L, Cipollone F, Santovito D, Carpineto P and Mastropasqua L: Role of microRNAs in the modulation of diabetic retinopathy. Prog Retin Eye Res 43: 92-107, 2014.

35. Chen CF, Hua K, Woung LC, Lin CH, Chen CT, Hsu CH, Liou SW and Tsai CY: Expression profiling of exosomal miRNAs derived from the aqueous humor of myopia patients. Tohoku J Exp Med 249: 213-221, 2019.

36. Wang $\mathrm{G}$, Wu B, Zhang B, Wang $\mathrm{K}$ and Wang $\mathrm{H}$ : LncRNA CTBP1-AS2 alleviates high glucose-induced oxidative stress, ECM accumulation, and inflammation in diabetic nephropathy via miR-155-5p/FOXO1 axis. Biochem Biophys Res Commun 532: 308-314, 2020.

37. Assmann TS, Recamonde-Mendoza M, Puñales M, Tschiedel B, Canani LH and Crispim D: MicroRNA expression profile in plasma from type 1 diabetic patients: Case-control study and bioinformatic analysis. Diabetes Res Clin Pract 141: 35-46, 2018.

his work is licensed under a Creative Commons

Attribution-NonCommercial-NoDerivatives 4.0 International (CC BY-NC-ND 4.0) License. 